\title{
The Effect of Gesture-Command Pairing Condition on Learnability when Interacting with TV
}

\author{
Chun Ik Jo ${ }^{1}$, Ji Hyoun Lim ${ }^{1}$, Jun Park ${ }^{2}$ \\ ${ }^{1}$ Department of Industrial Engineering, Hongik University, Seoul, 121-791 \\ ${ }^{2}$ Department of Computer Engineering, Hongik University, Seoul, 121-791
}

\begin{abstract}
Objective: The aim of this study is to investigate learnability of gestures-commands pair when people use gestures to control a device. Background: In vision-based gesture recognition system, selecting gesture-command pairing is critical for its usability in learning. Subjective preference and its agreement score, used in previous study(Lim et al., 2012) was used to group four gesture-command pairings. To quantify the learnability, two learning models, average time model and marginal time model, were used. Method: Two sets of eight gestures, total sixteen gestures were listed by agreement score and preference data. Fourteen participants divided into two groups, memorized each set of gesture-command pair and performed gesture. For a given command, time to recall the paired gesture was collected. Results: The average recall time for initial trials were differed by preference and agreement score as well as the learning rate $\mathrm{R}$ driven by the two learning models. Conclusion: Preference rate agreement score showed influence on learning of gesture-command pairs. Application: This study could be applied to any device considered to adopt gesture interaction system for device control.
\end{abstract}

Keywords: Vision-based gesture interaction, One-hand gesture, Learning curve, Agreement score, Subjective preference

\section{Introduction}

인간과 기계 사이에서의 상호작용이란 입출력 장치를 매 개로 하여 컴퓨터와 사람이 주고 받는 일련의 의사소통 과정을 뜻한다(Kim, 2005). WIMP(Window, Icon, Menu, Pointer)로 대변되던 GUI 시대에는 키보드와 마우스가 대 표적인 상호작용 수단이었으나, 센서 및 통신기술의 발전으 로 새로운 상호작용 방식이 등장하였다. 음성, 촉감, 시점, 제스처 등에 기반한 새로운 사용자 인터페이스가 개발되고 있으며 (Hong \& Woo, 2008), 이러한 상호작용 수단 중 제 스처 기반 사용자 인터페이스는 부가적인 장비 사용 부담 이 적고 사용자의 자유로운 의사 전달이 가능하다는 이점 이 있어 이에 관한 연구가 활발히 진행되고 있다(Kim et al., 2010).

Nielsen(1993)은 사용자가 시스템을 효율적으로 사용 할 수 있는지를 측정하는 지표들 중의 하나로 학습성 (learnability)을 제안하였다. 그리고 학습성의 평가는 학습 곡선을 사용한 수행도의 향상, 회상 및 재인을 이용한 기 억의 측정 등이 사용될 수 있다고 제안하였다. 제품의 사용 과 관련하여 학습성의 개념을 도입한 연구(Jung \& Hong, 2008) 에서는 제품의 반복적인 사용에서 측정된 작업수행 시간을 토대로 학습성을 평가하여, 학습률을 통한 제품의 사 용편의성 분석방법을 제안하였다.

본 연구에서는 Kincet를 이용한 비전 기반 제스처 인식 시스템을 사용하여 TV를 제어하는 상황을 연구 대상으로 한다. TV를 제어하기 위하여 비접촉식 제스처를 사용하는 경우, 사용자는 TV가 동작하기 원하는 명령 (command)을

Corresponding Author: Ji Hyoun Lim. Department of Industrial Engineering, Hongik University, Seoul, 121-791.

Phone: +82-2-320-3072, E-mail: limjh@hongik.ac.kr

Copyright@2012 by Ergonomics Society of Korea(pISSN:1229-1684 eISSN:2093-8462). All right reserved.

(c) This is an open-access article distributed under the terms of the Creative Commons Attribution Non-Commercial License(http://creativecommons.org/licenses/by-nc/3.0/), which permits unrestricted non-commercial use, distribution, and reproduction in any medium, provided the original work is properly cited. http://www.esk.or.kr 
적합한 동작(gesture)수행을 통하여 TV와 상호작용하는 환 경으로 이루어진다. 이 경우, command와 gesture를 연관하 는 방법에 따라 각각의 gesture-command 쌍의 학습성이 다를 것이라 예상된다.

비전 기반 동작 인식 시스템 환경을 가정하여 진행한 선 행연구(Lim, Jo \& Kim, 2012)에서 39가지 gesture와 30 가지 command를 사용하여 도출한 1,170 개의 gesturecommand 쌍에 대하여 30명의 실험참여자로부터 주관적인 선호도를 수집하였고, 이를 통해 gesture-command를 연 관시키는 과제에서 의견일치도가 도출되었다. 이를 바탕으 로 gesture-command 쌍에 대한 평균적인 선호도 및 의견 일치도가 gesture-command 쌍의 학습에 미치는 영향에 대해 알아보고자 한다.

\section{Method}

\subsection{Experiment hypothesis}

본 연구는 gesture-command 쌍의 특성이 학습에 미 치는 영향에 대하여 실험을 통해 알아보는 것을 목적으로 한다. 일반적으로 사람들이 선호하는 gesture-command 쌍이라면 command를 보고 gesture를 떠올리는 시간이 짧 을 것이며, 학습이 빨리 이루어질 것이라고 기대할 수 있다. 의견일치도가 높은 gesture-command 쌍의 경우도 마찬 가지로 command를 보고 gesture를 떠올리는데 걸리는 시 간이 짧고, 학습이 용이할 것이라고 예상할 수 있다. 따라서 gesture-command 쌍의 요소인 주관적 선호도와 의견일 치도가 원하는 command를 실행하기 위해 수행해야 하는 gesture를 떠올리는데 걸리는 시간에 영향을 미치는지를 알 아보기 위하여 다음과 같은 실험을 설계하였다.

실험을 위한 독립변수는 gesture-command 쌍 각각의
선호도 점수와 의견일치도이고, 종속변수는 주어진 command 를 실행하기 위해 수행해야 하는 gesture를 떠올리는데 걸 리는 시간(recall time) 과 반복 수행에서 얻어지는 학습률이 다. 실험을 통해 검증하고자 하는 가설은 다음 두 가지이다.

H1: 평균적인 선호도가 높은 gesture-command 쌍과 그 렇지 않은 쌍은 command를 수행하기 위한 gesture를 떠올리는데 걸리는 시간이 다를 것이고, 학습성도 다를 것이다.

H2: 의견일치도가 높은 gesture-command 쌍과 그렇지 않은 쌍은 command를 수행하기 위한 gesture를 떠 올리는데 걸리는 시간이 다를 것이고, 학습성도 다를 것이다.

\subsection{Selection criteria of gesture-command pairing}

본 연구에서 사용한 command-gesture list는 선행연구 (Lim et al., 2011)에서 사용한 39개의 gesture 중 오른손 만을 사용한 것으로 제한하고, 이 중 의견일치도를 기준으로 상위 $25 \%$ 와 하위 $25 \%$ 항목을 선정하였다. 의견일치도 역 시 선행연구에서 39 개의 gesture가 각각 주어졌을 때 30 개 의 command 항목들 중 1순위로 연상된 command 항목을 기준으로 Wobbrock et al.(2009)이 제안한 식을 이용하여 계산하였으며, 계산식은 (1) 과 같다.

$$
A_{r}=\sum_{P_{i} \subseteq P_{r}}\left(\frac{\left|P_{i}\right|}{\left|P_{r}\right|}\right)^{2}
$$

$$
\begin{aligned}
& A_{r}: \text { Gesture } r \text { 에 대한 의견일치도 } \\
& P_{r}: \text { Size of command pool }=30 \\
& P_{i}: \text { Command }{ }_{i} \text { 로 의견이 일치한 수 }
\end{aligned}
$$

\begin{tabular}{|c|c|c|c|c|}
\hline & & & \multicolumn{2}{|c|}{ Gesture-command preference } \\
\hline & & Gesture list(Agreement Score) & Good & $\mathrm{Bad}$ \\
\hline \multirow{2}{*}{$\begin{array}{l}\text { Agreement } \\
\text { score }\end{array}$} & High & $\begin{array}{c}\text { 우측하단에서 위로 이동 }(0.3089) \\
\text { 좌측상단에서 우로 이동 }(0.2533) \\
\text { 우측상단에서 아래로 이동( }(0.2600) \\
\text { 주먹 모양 }(0.1378)\end{array}$ & $\begin{array}{l}\text { Volume } \operatorname{Up}(57) \\
\text { 채널 간 이동(53) } \\
\text { Volume Down(48) } \\
\quad \text { 음소거(33) }\end{array}$ & $\begin{array}{l}\text { PIP(Dual View) 닫기(1) } \\
\text { 선호 채널 해제(1) } \\
\text { Home(1) } \\
\text { 선호 채널 간 이동(1) }\end{array}$ \\
\hline & Low & $\begin{array}{c}\text { 우측하단에서 좌측상단방향 사선이동 }(0.0867) \\
\text { 좌측상단 }- \text { 우측상단 }- \text { 우측하단 }- \text { 좌측하단 이동 }(0.0933) \\
\text { 우측상단에서 하단 - 좌측방향 직각이동 }(0.0689) \\
\text { 좌측상단에서 우측 - 하단방향 직각이동 }(0.0689)\end{array}$ & $\begin{array}{c}\text { Home/이전 메뉴로 복귀(16) } \\
\text { 전체 화면(24) } \\
\text { Menu 닫기(16) } \\
\text { Menu 호출(16) }\end{array}$ & $\begin{array}{l}\text { 선택 방송 보기(1) } \\
\text { [VOD] forward(1) } \\
\text { [VOD] backward(1) } \\
\text { 화면 잠금 해제(1) }\end{array}$ \\
\hline
\end{tabular}

Table 1. Selected command \& gesture list 
각각의 gesture에 짝지어지는 command는 선행연구에 서 수집한 정보 중 주관적인 선호도 점수가 가장 높은 것 과, 주관적인 선호도 점수가 1 이상 중 가장 낮은 것을 기준 으로 선정하였으며, 선정한 결과는 Table 1 과 같다. 이때, gesture-command 쌍에 중첩이 생기지 않도록 gesture와 command 쌍을 선택하였다.

그 결과 의견일치도가 높은 그룹, 즉 하나의 gesture에 대해서 대부분의 사람들이 동일한 command를 연상하는 그룹은 '우측하단에서 위로 이동', '좌측상단에서 우로 이동', '우측상단에서 아래로 이동' 그리고 '주먹모양'으로 구성되었 고, 의견일치도가 낮은 그룹, 즉 gesture에 대하여 몇 가지 다른 command가 연상되는 그룹은 '우측하단에서 좌측상 단 방향으로 사선 이동', '좌측상단에서 우측상단으로, 다시 우측하단으로, 좌측하단으로 이동', '우측상단에서 하단, 그 리고 좌측방향을 직각 이동' 그리고 '좌측상단에서 우측으 로, 그리고 하단방향으로 직각이동'으로 구성되었다. 이 각 각의 gesture들에 대해서 주관적선호도가 높은 gesturecommand 쌍과 선호도가 낮은 gesture-command 쌍이 각 각 선정되었다. 이 경우, 동일한 gesture를 다른 command 와 쌍을 이루기 때문에, 실험 자극은 counter balanced된 두 종류의 세트가 아래와 같이 준비되었다.

Set $A$ : 총 8쌍의 gesture-command 쌍

- High agreement score \& Bad pairing $(\mathrm{BH}) 4$ 쌍

- Low agreement score \& Good pairing (GL) 4쌍

Set B: 총 8쌍의 gesture-command 쌍

- High agreement score \& Good pairing (GH) 4쌍

- Low agreement score \& Bad pairing(BL) 4쌍

\subsection{Experimental environment \& apparatus}

실험장비는 C\#프로그램을 기반으로 Kinect를 활용하여 개발하였다. 영상출력위치에서는 실험참여자의 모습이 거울 상의 형태로 제시되며, 장비는 오른손의 손끝 궤적정보를 수 집한다. 실험참여자는 Kinect에서부터 전방으로 $2 \mathrm{~m}$ 거리의 정해진 위치에 서서, 영상출력위치에 표시되는 화면을 보면 서 동작을 수행한다.

실험진행자는 별도의 모니터를 통해 프로그램을 제어한다. 실험에 사용될 command list를 입력한 뒤 프로그램을 시작 하면, 실험참여자에게 거울상 화면을 제시하게 된다. 거울상 화면에서는 실험진행자의 오른손 위치에 별모양의 visual feedback을 제공하였다. 실험진행자만 볼 수 있는 화면에서 'Start' 버튼을 누르면 실험참여자가 보는 화면 상단 중앙에 Ready, 3, 2, 1을 순차적으로 0.5초씩 보여준 뒤 입력된 command 중 하나가 무작위로 표시된다. 실험참여자는 주 어진 command를 실행하는 gesture를 기억해 내면, 왼손에 휴대중인 무선 포인터 장비의 버튼을 누름으로써 동작의 시 작을 알린 뒤, 오른손으로 gesture를 수행하고 다시 왼손에 휴대중인 장비의 버튼을 눌러 수행 종료를 알린다.

Figure 1에 도시된 바와 같이, 실험진행자는 실험참여자의 gesture가 적절하게 수행되었을 경우 'Correct'로, 그렇지 않을 경우 'Error'로 판정하며, 'Correct'일 경우에만 수행정 보를 수집한다. 만일 'Error'로 판정될 경우, 현재 수행중인 command의 오류횟수가 1 씩 누적되며, 해당 command는 수행하지 않은 상태로 간주되고 이후 남아있는 command list에 추가된 후 무작위로 실험참여자에게 제시된다. 실험참 여자가 적합한 gesture를 기억해 내고, 수행 전 무선 포인 터의 버튼을 누를 때까지의 시간(recall time), gesture 수 행 후 버튼을 다시 눌러 종료할 때까지의 시간(movement time)을 msec 단위로 기록하였다.

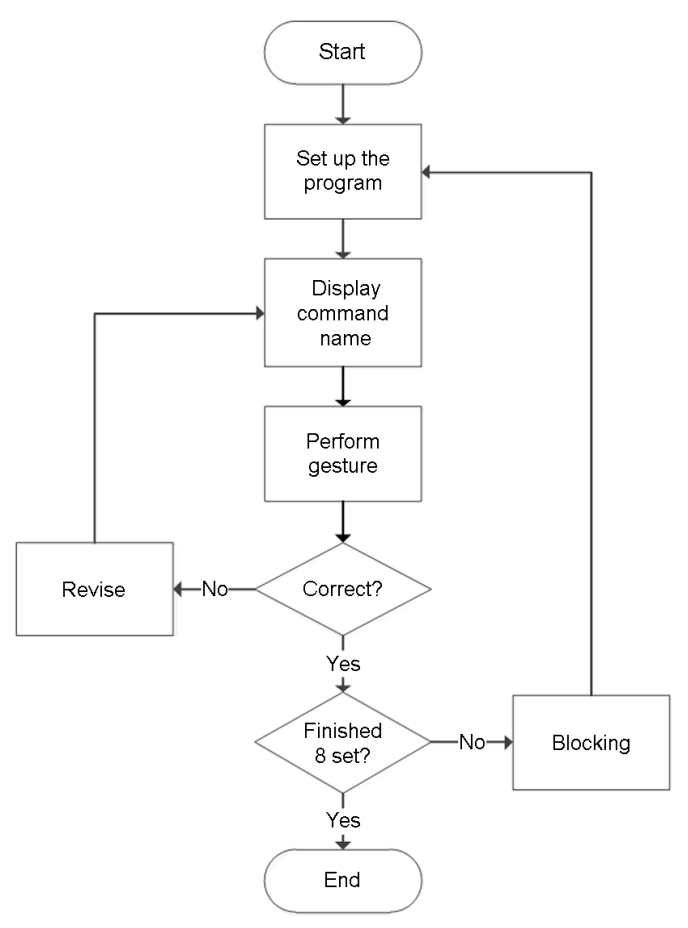

Figure 1. Flow chart for experiment application

\subsection{Participants}

총 14 명의 실험참여자(남성 10 명, 여성 4 명)가 본 실험에 참여하였으며, 이들의 평균 연령은 24.6세였다. 실험참여자 중 남성 7명 $(70 \%)$, 여성 3명 $(75 \%)$ 가 Kinect나 Wii 등 제 스처 기반 인터페이스를 사용해 본 경험이 있었으나, TV를 


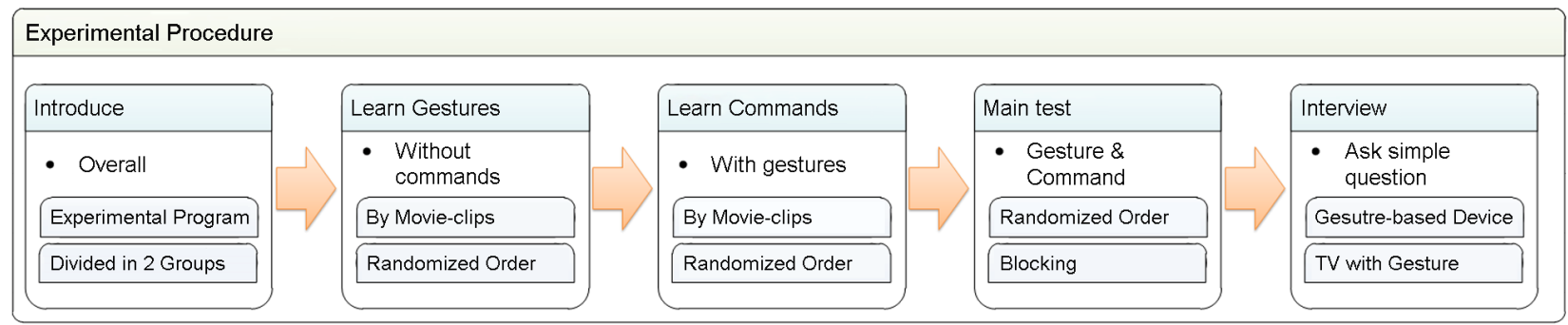

Figure 2. Experimental procedure

비접촉식 제스처로 조작해 본 경험이 있는 사람은 없었으며, 실험참여자 중 1 명을 제외하고 모두 오른손잡이였다. 선정한 gesture-command 쌍은 실험참여자를 2개의 그룹으로 나 누어 참여 순서에 따라 gesture-command set $\mathrm{A}$ (8개의 gesture-command 쌍) 와 set B(8개의 gesture-command 쌍)를 교대로 제시하였다.

\subsection{Experimental procedure}

실험절차는 Figure 2에서 보여주는 바와 같이 실험소개, gesture 학습, 본 실험 및 사후인터뷰로 진행되었다. 실험소 개 단계는 실험의 목적과 방법에 대해 실험참여자에게 설 명하는 단계로, 실험참여자가 동작을 입력하는 방식을 안내 하였다.

실험소개 단계 이후에 진행한 gesture 학습 단계에서는 Table 1에 제시된 8종류의 gesture list를 암기시키기 위한 단계로, 실험참여자에게 동영상을 통해 손동작을 제시한 뒤 기억나는 순서에 따라 무작위로 수행하도록 하고 모두 맞게 수행할 때까지 진행하였다.

본 실험 단계에서는 set A 또는 set B에 속하는 8쌍의 gesture-command 쌍을 제시하고, 주어진 set를 학습하도 록 하였다. 실험참여자는 command와 gesture의 연관을 숙 지할 때까지 동영상을 반복하여 보았다. 최소 3 회 이상 반복 시청 후, 추가 시청은 실험참여자의 요청에 의해 이루어졌다. 이후, 실험참여자는 무작위 순서로 제시되는 command에 해당하는 gesture를 수행하도록 하였다. Command에 대해 적절한 gesture를 수행하지 못했을 경우 'Error'로 판정하
고, 올바른 gesture-command 쌍을 다시 학습시켜 수행하 도록 하였다. 8쌍의 gesture-command를 연속으로 성공하 면, 휴식시간을 제공하였고 휴식시간 동안 구구단을 외우도 록 하였다.

본 실험 종료 후, 사후인터뷰 단계에서는 간단한 개인정보 및 제스처 기반 장비나 Smart TV 사용 경험에 대한 정보를 수집하였다.

\section{Results}

Gesture-command 쌍의 요소인 주관적 선호도와 이에 대한 의견일치도에 따라 4 개의 분류로 분류한 뒤, 수행 순서 에 따라 recall time의 평균값을 계산한 결과는 Table 2 와 Figure 3 와 같다. 이 결과를 토대로 gesture-command 쌍의 특성에 따라 recall time의 차이가 있는지 여부를 알 수 있다.

Gesture-command 쌍을 맞게 수행했을 때의 결과값을 토대로, 각 분류 별 초기평균 recall time의 크기는 $\mathrm{GL}$ (2.8365) $>\mathrm{BH}(2.5439)>\mathrm{BL}(1.5159)>\mathrm{GH}(1.0758)$ 순으 로 나타났다. 실험이 진행될수록 수행 순서에 따른 차이가 존재했으나, 초기평균 recall time보다 낮아지는 경향을 보 였다. $\mathrm{GH}$ 의 초기평균 recall time이 가장 낮게 도출되었으 며, GL의 초기평균 recall time이 가장 크게 나타났다.

초기평균 recall time에 대한 분석을 실시하기 위해서 1 회 차 수행에 대한 데이터를 정리하면 Table 3 과 같다.

Table 2. Average recall time in 4 categories

\begin{tabular}{c|c|c|c|c|c|c|c|c|c|c|c}
\hline 반복수 & 1회 & 2회 & 3회 & 4회 & 5회 & 6회 & 7회 & 8회 & 9회 & 10회 & 11회 \\
\hline $\mathrm{GH}$ & 1.0758 & 0.8906 & 1.0197 & 0.9349 & 1.0125 & 0.9904 & 1.0745 & 1.0335 & 0.9990 & 1.0488 & 1.1193 \\
\hline $\mathrm{GL}$ & 2.8365 & 2.1695 & 1.8467 & 1.7511 & 1.4210 & 1.2849 & 1.2982 & 1.3005 & 1.3255 & 1.1830 & 1.6054 \\
\hline $\mathrm{BH}$ & 2.5439 & 1.8471 & 1.5285 & 1.5074 & 1.3697 & 1.2638 & 1.4600 & 1.3169 & 1.2812 & 1.1279 & 1.2616 \\
\hline $\mathrm{BL}$ & 1.5159 & 1.1245 & 1.1730 & 1.3860 & 1.2760 & 1.2188 & 1.3823 & 1.2793 & 1.4596 & 2.1168 & 1.6388 \\
\hline
\end{tabular}




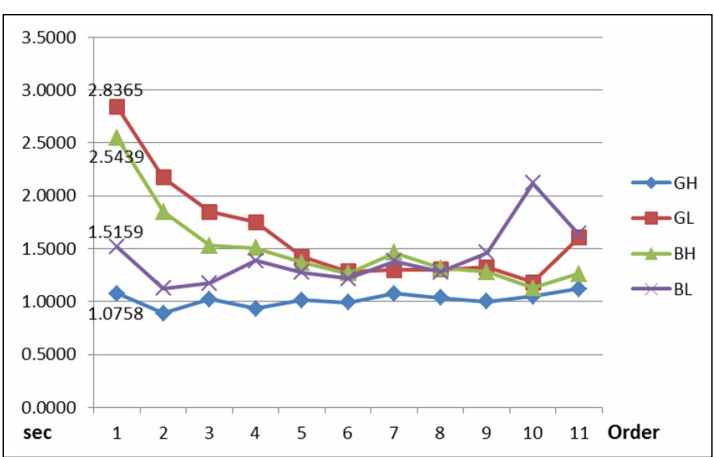

Figure 3. Recall times trend for 4 categories

Table 3. Summary of initial recall time

\begin{tabular}{c|c|c|c}
\hline \multicolumn{2}{c|}{$\begin{array}{c}\text { Mean } \\
(\mathrm{SD})\end{array}$} & \multicolumn{2}{c}{ Gesture-command preference } \\
\cline { 3 - 4 } & & Good & Bad \\
\hline \multirow{2}{*}{$\begin{array}{c}\text { Agreement } \\
\text { score }\end{array}$} & High & $1.0758(0.0782)$ & $2.5439(1.6581)$ \\
\cline { 2 - 4 } & Low & $2.8365(1.5450)$ & $1.5159(0.2600)$ \\
\hline
\end{tabular}

\section{Analysis}

\subsection{Initial recall time}

14 명의 실험참여자들이, 실험소개 및, gesture 학습 단 계를 거처 본 실험에서 수집된 초기 recall time을 사용하 여, 그룹 간의 차이를 검증하여 보았다. 4 가지 그룹 $(\mathrm{GH}$, $\mathrm{GL}, \mathrm{BH}, \mathrm{BL})$ 간에 통계적으로 유의한 차이가 있었으나, 선호도 단독 $(\mathrm{G \#}$ versus $\mathrm{B \# )}$ 또는 의견일치도 단독(\#H versus \#L)의 영향은 통계적으로 유의하지 않았다. 한편, 실험 set $\mathrm{A} / \mathrm{B}$ 의 차이는 통계적으로 유의한 것으로 나타났 다 $(p<0.001)$.

\subsection{Average time model}

학습곡선은 평균시간모델(Average Time Model) 과 한계 시간모델 (Marginal Time Model)의 두 가지 수학적 모델로
정의할 수 있다. $\mathrm{R}$ 을 학습률이라고 할 때, 평균시간모델은 생산량이 두 배로 증가될 때 누적평균생산시간이 (1-R)만 큼 감소되는 것을, 한계시간모델은 생산량이 두 배로 증가될 때마다 단위생산시간이 $(1-\mathrm{R})$ 만큼 감소한다는 것을 가정한 다(Hwang, 1987). 본 연구에서는 학습곡선의 2가지 모델을 적용하여 gesture-command 쌍의 특성이 학습에 어떤 영 향을 미치는지 알아보고자 하였다.

평균시간모델은 학습이 진행될수록 누적평균시간이 (1-R) 만큼 감소한다는 것을 가정한다. 평균시간모델을 본 연구에 서는 다음과 같이 적용하였다.

$$
\begin{aligned}
& Y_{x}=\mathrm{a} R^{m} \\
& x=2^{m}
\end{aligned}
$$

$Y_{x}: x$ 번째 수행에서의 누적평균 recall time

$\mathrm{a}$ : 첫 번째 수행에서의 평균 recall time

$m$ : 수행 수가 두 배로 증가된 횟수

$x$ : 반복 수행 횟수

$\mathrm{R}$ : 학습률

이 식을 정리하면 다음의 식을 얻을 수 있다.

$$
Y_{x}=a X^{b}
$$

실험에서 측정한 recall time을 평균시간모델에 준하여 4 가지 분류 별로 해당 수행 시기에 따라 예측한 결과는 Table 4 과 Figure 4 과 같다. 평균시간모델에 근거하여 $\mathrm{R}$ 값을 산정한 결과, 각 분류는 $\mathrm{GH}(0.9139)>\mathrm{GL}(0.8824)$ $>\mathrm{BL}(0.8709)>\mathrm{BH}(0.8630)$ 의 크기 순으로 나타났다. 산 정한 R값을 토대로 계산식 (2)를 통해 각 분류 별 누적 평균 recall time을 예측하면 $\mathrm{GH}$ 의 누적평균 recall time 은 $Y_{x}=1.0758 X^{-0.1298}$ 로, $\mathrm{GL}$ 의 누적평균 recall time은 $Y_{x}$ $=2.8365 X^{-0.1905}$ 로, $\mathrm{BH}$ 의 누적평균 recall time은 $Y_{x}=$ $2.5439 X^{-0.2125}$ 로, $\mathrm{BL}$ 의 누적평균 recall time은 $Y_{x}=$ $1.5159 X^{-0.1994}$ 로 각각 계산할 수 있다.

이를 기초로 각 수행 시의 평균 recall time 예측값은 수 행 시기 별로 예측한 누적평균시간에서 이전 수행까지의 예 측값을 감하여 산출할 수 있다.

Table 4. Predicted average recall time by the Average Time Model

\begin{tabular}{c|c|c|c|c|c|c|c|c|c|c|c}
\hline 수행 & 1회 & 2회 & 3회 & 4회 & 5회 & 6회 & 7회 & 8회 & 9회 & 10회 & 11 회 \\
\hline $\mathrm{GH}$ & 1.0758 & 0.8906 & 0.8319 & 0.7960 & 0.7703 & 0.7504 & 0.7342 & 0.7207 & 0.7091 & 0.6989 & 0.6899 \\
\hline $\mathrm{GL}$ & 2.8365 & 2.1695 & 1.9732 & 1.8557 & 1.7729 & 1.7096 & 1.6587 & 1.6163 & 1.5802 & 1.5487 & 1.5210 \\
\hline $\mathrm{BH}$ & 2.5439 & 1.8471 & 1.6518 & 1.5365 & 1.4561 & 1.3951 & 1.3463 & 1.3059 & 1.2715 & 1.2418 & 1.2156 \\
\hline $\mathrm{BL}$ & 1.5159 & 1.1245 & 1.0125 & 0.9461 & 0.8995 & 0.8641 & 0.8357 & 0.8121 & 0.7921 & 0.7747 & 0.7594 \\
\hline
\end{tabular}




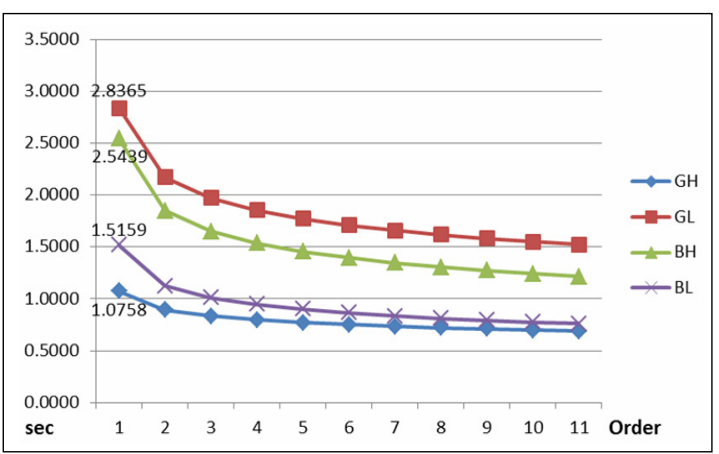

Figure 4. Predicted averages of recall time by Average Time Model

\subsection{Marginal time model}

평균시간모델은 학습이 진행될수록 단일평균시간이 $(1-\mathrm{R})$ 만큼 감소한다는 것을 가정한다. 한계시간모델을 본 연구에 서는 다음과 같이 적용하였다.

$$
\begin{aligned}
& U_{x}=\mathrm{a} R^{m} \\
& x=2^{m}
\end{aligned}
$$

$U_{x}: x$ 번째 수행에서의 누적평균 recall time

$\mathrm{a}$ : 첫 번째 수행에서의 평균 recall time

$m$ : 수행 수가 두 배로 증가된 횟수

$x$ : 반복 수행 횟수

$\mathrm{R}$ : 학습률

따라서 $x$ 번 반복 수행했을 때의 단일평균 recall time $U_{x}$ 는 다음의 계산식으로 유추할 수 있다.

$$
U_{x}=\mathrm{a} x^{b}
$$

실험에서 측정한 recall time을 한계시간모델에 준하여 4 가지 분류 별로 해당 수행 시기 별 예측결과는 Table 5 와 Figure 5 과 같다. 한계시간모델에 근거하여 R값을 산정한 결과, 각 분류 별 R값의 크기는 $\mathrm{GH}(0.8279)>\mathrm{GL}$ (0.7649)
$>\mathrm{BL}(0.7418)>\mathrm{BH}(0.7261)$ 의 순으로 나타났다. 산정한 $\mathrm{R}$ 값을 토대로 계산식 (3)를 통해 각각의 분류 별 단일평 균 recall time을 예측하면 $\mathrm{GH}$ 의 단일평균 recall time은 $U_{x}=1.0758 x^{-0.2725}$ 로, $\mathrm{GL}$ 의 단일평균 recall time은 $U_{x}=$ $2.8365 x^{-0.3867}$ 로, $\mathrm{BH}$ 의 단일평균 recall time은 $U_{x}=$ $2.5439 x^{-0.4618}$ 로, $\mathrm{BL}$ 의 단일평균 recall time은 $U_{x}=$ $1.5159 x^{-0.4 .309}$ 로 각각 계산할 수 있다.

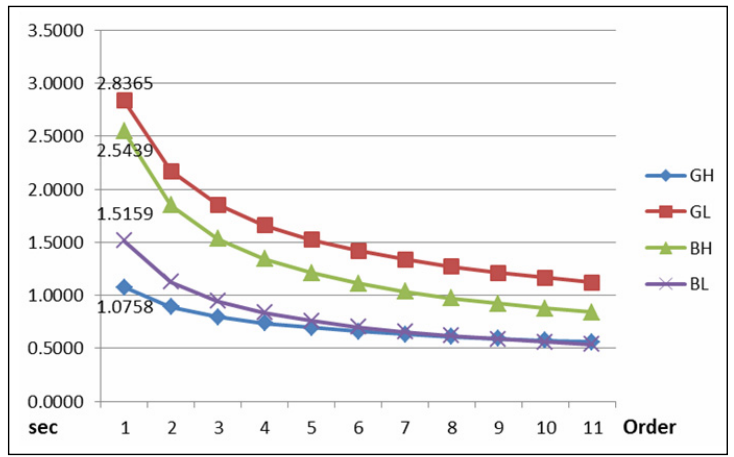

Figure 5. Predicted average of recall time by Marginal Time Model

학습곡선의 두 모델에 근거하였을 때의 R값은 $\mathrm{GH}>\mathrm{GL}>$ $\mathrm{BL}>\mathrm{BH}$ 의 크기 순으로 나타났다. 주관적인 선호도가 good 인 경우, 의견일치도가 높을 경우의 학습률 R값이 더 컸으 며, 선호도가 bad인 경우, 의견일치도가 낮을 경우의 R값 이 낮게 계산되었다.

\section{Discussion}

본 연구는 비접촉 동작 인식 시스템 하에서 gesturecommand 쌍의 학습에 대한 연구로서, gesture-command 쌍에 대한 선호도와 의견일치도가 학습에 어떠한 영향을 미치는지를 알고자 하였다. 의도하는 기기의 조작을 위해, 해당 command를 기기에 전달하기 위한 적합한 gesture를

Table 5. Predicted average recall time by the Marginal Time Model

\begin{tabular}{c|c|c|c|c|c|c|c|c|c|c|c}
\hline 수행 & 1 회 & 2회 & 3회 & 4회 & 5회 & 6회 & 7회 & 8회 & 9회 & 10회 & 11 회 \\
\hline $\mathrm{GH}$ & 1.0758 & 0.8906 & 0.7974 & 0.7373 & 0.6938 & 0.6602 & 0.6330 & 0.6104 & 0.5911 & 0.5744 & 0.5596 \\
\hline $\mathrm{GL}$ & 2.8365 & 2.1695 & 1.8547 & 1.6594 & 1.5222 & 1.4185 & 1.3364 & 1.2692 & 1.2127 & 1.1642 & 1.1221 \\
\hline $\mathrm{BH}$ & 2.5439 & 1.8471 & 1.5317 & 1.3412 & 1.2099 & 1.1122 & 1.0358 & 0.9738 & 0.9223 & 0.8785 & 0.8406 \\
\hline $\mathrm{BL}$ & 1.5159 & 1.1245 & 0.9442 & 0.8341 & 0.7577 & 0.7004 & 0.6554 & 0.6188 & 0.5881 & 0.5620 & 0.5394 \\
\hline
\end{tabular}


떠올리는데 걸리는 시간인 recall time을 학습곡선의 두 가 지 모형으로 분석하였다.

실험으로 도출된 결과의 초기평균 recall time의 크기가 모두 다르나, 반복 수행할수록 그 차이가 점차 감소하는 모 습을 보였다. 선호도가 good인 경우에는 의견일치도가 높을 경우 초기평균 recall time이 낮았으며, 선호도가 bad인 경 우에는 의견일치도가 높을 경우 초기평균 recall time이 높 았다. 학습률의 측면에서 살펴보면, 선호도가 good인 경우 에는 의견일치도가 높을 경우 학습률 R값이 높았으며, 선호 도가 bad인 경우에는 의견일치도가 높을 경우 학습률 R값 이 낮았다.

학습률 분석결과, $\operatorname{Set} A(B H \& G L)$ 와 $\operatorname{Set} B(G H \& B L)$ 간의 차이도 두드러지게 나타났다. 이는 조건으로 제시된 gesture-command 쌍 자체의 성격만큼이나 학습대상 list 가 어떻게 구성되어 있는가도 영향을 미친다는 것을 의미한 다. 즉, $\mathrm{BL}$ 그룹의 낮은 초기평균 recall time과 높은 학습률 $\mathrm{R}$ 값을 보인 것은, $\mathrm{GH}$ 그룹과 같은 자극 set에 포함되어 제 시되었기 때문으로 추측된다 이러한 점을 검증하기 위해서 는 4 가지 group을 별도의 실험 세트로 구성하는 추가 연구 가 필요하다.

이러한 결과는 향후 비접촉 제스처 인터랙션 시스템에 서 사용자가 TV를 제어하는 환경에서 수행할 수 있는 gesture-command 쌍을 선정하는데 활용할 수 있을 것으 로 예상된다. 주관적인 선호도와 의견일치도에 대한 조사는 gesture 사용에 있어서 회상이 용이한 gesture-command 쌍을 설계하는데 지표로 활용될 수 있다. 그리고 만일 주관 석인 선호도나 의견일치도가 열등한 경우라도, 10 회 이상의 반복 수행을 통해 회상시간이 줄어들고 초기 회상시간의 차 이를 크게 줄일 수 있다는 점도 gesture-command 쌍 설 계에서 고려될 수 있을 것이다.

\section{Acknowledgements}

This work was supported by 2012 Hongik University Research Fund.

\section{References}

Hong., D. \& Woo, W., H., Recent research trend of gesture-based user interface, Telecommunications Review, 18(3), 403-413, 2008.

Hwang, H., Work Management Theory, Youngchi Publishers, Revised and enlarged edition, 1987.

Jung, K. T. \& Hong, J. I., Application of learning curve to evaluate product learnability, Journal of the Ergonomics Society of Korea, 27(2), 59-65, 2008.

Kim, J. W., Introduction to Human Computer Interaction, Ahn Graphics, 2005.

Kim, Y. W., Whang, M. C., Kim, J. H., Woo, J. C., Kim, C. J. \& Kim, J. H., Analysis of $3 \mathrm{D}$ motion recognition using meta-analysis for interaction, Journal of the Ergonomics Society of Korea, 29(6), 925 $-932,2010$.

Lim, J. H., Jo, C. I. \& Kim, J., "Implicit Associations between Gesture and Commands", proceedings of the Spring Conference on Ergonomics Society of Korea, Jeju, 2012.

Nielson, J., Usability Engineering, Morgan Kaufmann Publishers, 1993.

Wobbrock, J. O., Morris, M. R. and Wilson, A. D., "User Defined Gestures for Surface Computing", Proceedings of the Conference on Human Factors in Computing Systems, 2(pp. 1083-1092), Boston, MA, 2009.

\section{Author listings}

Chun Ik Jo: Chunikjo@gmail.com

Highest degree: BSE, Industrial Engineering, Hongik University Position title: Graduate student, Department of Industrial Engineering, Hongik University

Areas of interest: User Experience Analysis

Ji Hyoun Lim: limjh@hongik.ac.kr

Highest degree: Ph.D., University of Michigan

Position title: Assistant Professor, Department of Industrial Engineering, Hongik University

Areas of interest: Computational Cognitive Modeling, User-Driven NPD

Jun Park: jpark@hongik.ac.kr

Highest degree: Ph.D., University of Southern California

Position title: Associate Professor, Department of Computer Science and Engineering, Hongik University

Areas of interest: Augmented Reality, Interactive Media, Gesture Recognition

Date Received : 2012-07-20

Date Revised :2012-07-27

Date Accepted : 2012-07-28 